\title{
Relación entre valoración de una situación y capacidad para enfrentarla
}

\section{Relationship between appraisal of situation and coping ability}

\author{
Ma. de Lourdes Rodríguez Campuzano ${ }^{1}$, Ireri Y. Ocampo Juárez y \\ Carlos Nava Quiroz \\ Facultad de Estudios Superiores Iztacala, Universidad Nacional Autónoma de México
}

(Recepción: Abril 2009 - Aceptación: Julio 2009)

\begin{abstract}
Resumen
El presente estudio parte de la necesidad de entender, desde una concepción naturalista, algunos de los aspectos que participan en el fenómeno denominado estrés/afrontamiento. Su objetivo fue evaluar la correspondencia funcional entre dos dimensiones de algunas competencias cotidianas: la dimensión efectiva, esto es, lo que hacen o dicen los participantes en situaciones que les demandan logro, y la dimensión valorativa, esto es lo que creen respecto de ellas. Se aplicó un instrumento para evaluar competencias a una muestra intencional voluntaria de 347 participantes de entre 15 y 70 años de edad. Los resultados mostraron una correlación baja entre las dimensiones efectiva y valorativa, lo que sugiere cierta autonomía funcional entre dichas dimensiones. Los participantes no fueron totalmente competentes, en términos efectivos, ante las situaciones muestreadas, aunque estos puntajes fueron mayores que los obtenidos para la dimensión valorativa, es decir, valoraban las situaciones como amenazantes con menor frecuencia. De la misma forma se encontraron diferencias de género, los puntajes obtenidos por los hombres fueron ligeramente superiores para ambas dimensiones. Los hallazgos se discuten en relación con el concepto de percepción de amenaza.

Palabras Clave: Estrés, afrontamiento, competencias, percepción de amenaza, dimensiones competenciales.
\end{abstract}

\begin{abstract}
The present study emerged from the interest to understand, from a naturalistic point of view, some of the issues related to stress/coping phenomena. Its objective was to evaluate functional correspondence between day to day competence dimensions: effective dimension, referred to what people do or say on goal featured situations and valuative dimension, referred to what people believe about those situations. An instrument to evaluate day to day competences was applied to 347 participants among 15 to 70 years old. Results showed a low correlation between effective and valuative dimensions suggesting some kind of functional independence between them. They also showed that participants reported that they were not completely capable, in an effective way, to deal with the situations sampled on the instrument, but, even so, their scores on this dimension were bigger than those obtained for valuative dimension, that is, they valuate situations as threatens on about half of the items. Additionally gender differences were found, scores on both dimensions were bigger for men than for women. Results are discussed considering the concept of perception of threatens.
\end{abstract}

Key words: Stress, coping, competences, threaten perception, competential dimensions.

1 Correspondencia a: Dra. Ma. de Lourdes Rodríguez Campuzano. Pekín \# 6, Jardines de Bellavista, Tlalnepantla, Edo. De Méx, C.P. 54050. E-mail: carmayu5@yahoo.com 


\section{Introducción}

En este trabajo se presenta el reporte de un estudio cuyo objetivo fue evaluar la relación entre la dimensión efectiva de las competencias de las personas al enfrentar cierto tipo de situaciones cotidianas y su dimensión valorativa, esto es, la correspondencia entre la capacidad, en términos de lo que hacen o dicen los participantes, en algunas situaciones cotidianas y lo que creen con respecto a ellas. La realización de este estudio responde al interés por entender, desde una perspectiva naturalista, algunos de los aspectos que participan en el fenómeno que se ha denominado estrés/afrontamiento. El trabajo forma parte de una línea de investigación cuyo interés general es comprender la influencia del comportamiento en el estado de salud/enfermedad de las personas, lo cual resulta cada vez más relevante, dadas las crecientes evidencias de la participación de factores psicológicos en las enfermedades (Caballo, Buela-Casals y Carrobles, 1996).

La ubicación histórico-temporal del término estrés puede encontrarse, según Cassidy (1999), en las ciencias físicas en el siglo XVII. Sin embargo, como Lazarus y Folkman (1986) explican, el término puede ser hallado a principios del siglo XIV, cuando era usado para referirse a dificultad o adversidad.

A lo largo del tiempo este concepto ha sido definido de tres maneras distintas: como estímulo, como respuesta y como interacción (Brannon y Feist, 2001), por lo que Rice (1999) comenta que existen al menos tres significados distintos que corresponden a tres diferentes modelos: el modelo de estímulo, el modelo de respuesta y el modelo transaccional.

El primero hace referencia a cualquier evento o estímulo ambiental que causa tensión. En este caso, estrés es algo externo y, según Rice (1999), es más apropiado hacer referencia a estresores. Menciona como ejemplo la carga de trabajo.

El segundo se refiere a una respuesta subjetiva. Ya Cannon en 1929 (en Rodríguez, 1995) señalaba que el estrés era una respuesta de alarma que perturbaba el equilibrio interno del organismo y suponía que esta ruptura del equilibrio interno estaba causalmente relacionada con la enfermedad.

De acuerdo con Hans Selye (1956) el estrés es una respuesta general del organismo ante cualquier estimulo o situación estresante, aunque enfatiza la reacción biológica global producida y afirma que "estrés no es meramente tensión nerviosa” (p.30), sino que es "la respuesta no específica del cuerpo a cualquier demanda realizada", también conocida como Síndrome de Adaptación General (p.27). El estrés es considerado por dicho autor como el común denominador de todas las respuestas adaptativas del cuerpo.

El modelo de respuesta refiere a la experiencia de la persona; por ejemplo, se dice que alguien sufre de estrés. Aquí se infiere una experiencia abstracta llamada estrés a partir de diversos síntomas como irritabilidad, falta de energía o dolores de cabeza. Estos incluyen síntomas conductuales, emocionales y físicos que son exhibidos como consecuencia de las demandas particulares de la vida. En general, se habla de la reacción física del cuerpo en respuesta a una demanda. La demanda promueve un despertar natural del cuerpo a un nivel superior de actividad. La función de estas reacciones físicas es probablemente apoyar conductual y psicológicamente los esfuerzos de afrontamiento (Rice, 1999).

Siguiendo con el modelo y de acuerdo con Cassidy (1999), se ha desarrollado un debate en referencia a que si lo que ocurre primero es lo emotivo o lo conductual. Dicho autor sostiene que, apegados al sentido común, lo que sucede primero es la emoción, pero la velocidad de la reacción es tal que aparece como si todo ocurriera junto. De la misma forma, Walter Canon y Phillip Bard (1932, en Cassidy, 1999) argumentan que las respuestas emocional y conductual son simultáneas.

El tercer modelo es el llamado transaccional, y sus principales representantes son Lazarus y Folkman (1986). Estos autores conciben el estrés no como un estímulo, una respuesta subjetiva o una reacción biológica, sino como un proceso. Su manera de aproximarse a este fenómeno resultó un parte aguas en el estudio del fenómeno. La mayor parte de los expertos toman como base su definición y mantienen la misma postura conceptual. 
Lazarus y Folkman (1986) mencionan que el estrés hace referencia a una relación existente entre persona y entorno y que la clave de esta transacción es la valoración personal de la situación psicológica. Sugieren también que para que se presente un proceso de estrés son importantes dos elementos: la valoración cognitiva y las estrategias de afrontamiento. Con respecto al primero, con el trabajo aportado por Lazarus se acepta que opera en un número de etapas. Tan pronto como el evento externo es detectado por la persona y el despertar fisiológico comienza, la valoración cognitiva entra en juego decidiendo si existe o no peligro. Lazarus y Folkman (1984) llamaron a esto valoración primaria, es decir, al reconocimiento de que existe un problema. Si el peligro existe, el proceso valorativo juega un rol decidiendo la respuesta, esto es, de acuerdo con ellos, valoración secundaria. Es en esta etapa que se supone se etiqueta la emoción. De la evaluación cognitiva dependen las respuestas de estrés, y ante ello, se emplean diversas estrategias de afrontamiento. El uso de éstas depende de numerosos factores como la salud y la energía, las creencias positivas (creer que son capaces de obtener las consecuencias deseadas), capacidad para resolver problemas, habilidades sociales (la capacidad de hacer que otras personas colaboren puede constituir una importante fuente del manejo del estrés), y apoyo social o sensación de aceptación.

Después de que una persona ha valorado la situación inicialmente, se forma una idea de su capacidad de controlar o afrontar el peligro, la amenaza o el desafío, impresión que se denomina valoración secundaria. Y finalmente se realiza una reevaluación en donde la situación anteriormente valorada adquiere otro carácter que el original, si el entorno cambia o la persona empieza a considerar la situación en forma distinta.

Un factor importante es la capacidad o incapacidad de afrontar una situación estresante. Lazarus y Folkman (1986) definieron esta capacidad como el cambio constante de los esfuerzos cognitivos y conductuales para responder a las demandas específicas de carácter externo y/o interno que se valoran como elementos que exceden los recursos de una persona (Brannon y Feist, 2001), y a esto es lo que se ha llamado afrontamiento.

De acuerdo con Lazarus y Folkman (1984), las aproximaciones tradicionales al concepto de afrontamiento emergieron de dos fuentes separadas y distintas: la experimentación animal y la literatura psicoanalítica del ego. En el modelo animal, el término afrontamiento se define usualmente como actos que controlan condiciones aversivas y, por ello, de manejo lento o activación. El énfasis se encuentra en conductas de evitación y escape.

El término afrontamiento fue adoptado por psicólogos en las décadas de 1960 y 1970 para referirse al combate, derrota o manejo de las situaciones estresantes de la vida (Lazarus, 2006).

Los primeros intereses teóricos con el concepto comenzaron con las ideas freudianas referentes a las formas de defensa en la integridad psicológica de las personas. Desde esta perspectiva, el concepto de afrontamiento tiene que ver con cognición, con la diferenciación entre un número de procesos que las personas usan para manejar relaciones problemáticas (Lazarus y Folkman, 1984).

Lazarus (2006) menciona que el afrontamiento efectivo permite a las personas mantener niveles de estrés moderados y vivir con ello, sin que exista daño físico o psicológico significativo. También comenta que, como proceso, tiene tres significados: el primero concierne a lo que la persona actualmente piensa o hace, en contraste con lo que la persona usualmente hace, haría o podría hacer; el segundo a lo que la persona piensa o hace actualmente y que es examinado en un contexto específico. Pensamientos y acciones de afrontamiento son siempre dirigidas hacia condiciones particulares. Para entender el afrontamiento y evaluarlo, es necesario conocer lo que la persona está afrontando. Mientras más específicamente sea definido el contexto, más fácil será vincular acciones o pensamientos particulares con una demanda contextual. El tercero implica que para hablar de un proceso de afrontamiento es necesario hablar de cambios en pensamientos y acciones de afrontamiento como un resultado de encuentros con situaciones estresantes (Lazarus y Folkman, 1984).

Rice (1999) menciona que las conductas de afrontamiento pueden ser externas (acciones) o internas (cognitivas), positivas o negativas, de aproximación (activo) o de evitación, directas o indirectas; teniendo como función primaria: el prevenir, eliminar o reducir el estrés. 
Diversas investigaciones han empezado a buscar las variables que pueden moderar la relación estrés/enfermedad, y desde años pasados ha surgido el afrontamiento como el mejor candidato en esta búsqueda de moderadores. Se dice que si los individuos afrontan efectivamente los problemas que enfrentan, serán capaces de reducir las consecuencias nocivas del estrés (Billings y Moos, 1981).

El interés por estudiar el estrés y los factores que están relacionados con el mismo, se deriva del impacto que tiene este fenómeno en la salud. Puede producir efectos directos (incrementando la vulnerabilidad del organismo), actuar como agente inductor (precipitando la aparición de trastornos al alterar el funcionamiento fisiológico general del organismo), generar comportamientos pocos saludables (como fumar, abuso de sustancias psicoactivas, insumo o trastornos de alimentación, entre otros), o funcionar como factor agravante de una enfermedad (Cassidy, 1999).

Como se puede apreciar, el estrés es un proceso complejo y, según la perspectiva transaccional, refleja una relación recursiva y dinámica entre a) demandas ambientales, b) recursos sociales e individuales para enfrentar dichas demandas, y c) la valoración del individuo de esta relación. De hecho, se sostiene que está determinado por la percepción e interpretación de un evento, así como por la evaluación de la propia habilidad para afrontarlo (Graig, 1993). Se dice que la habilidad para manejar los estresores depende de los recursos de afrontamiento disponibles en la persona. Estos recursos pueden ser definidos a nivel biológico -sexo, condiciones físicas- y a nivel psicológico -personalidad, sentido del humor, asertividad- (Lazarus y Folkman, 1984).

Levy-Levoyer (1985) comenta que se deben considerar cuatro aspectos: 1) la condición de relación del estrés; 2) los estados psicológicos que no están ligados ni al sujeto ni al ambiente, sino a la inadecuación entre las necesidades y los recursos de los sujetos; 3) las características del ambiente; y 4) la importancia del significado de la situación para el individuo. Estos puntos permiten apreciar que, desde esta óptica, el significado personal dado por el sujeto a las situaciones ambientales es de gran importancia.

Lazarus y Launier (1978) sostienen que la característica determinante de la respuesta a un estresor es la valoración individual de la situación. Proponen que un evento puede ser analizado como pérdida (o daño), amenaza, o reto, y es la evaluación individual del evento lo que estimula un conjunto particular de reacciones de afrontamiento. El término amenaza es definido por estos autores como daño que es anticipado y que puede o no ser inevitable. Refieren también que la valoración realizada depende probablemente en parte de la configuración de los eventos ambientales en sí mismos. Así como de las creencias de la persona acerca del potencial de dominio. Lazarus y Folkman (1984), afirman que los procesos de evaluación cognitiva son, en gran medida, responsables de las variaciones en los sistemas de respuesta entre individuos bajo circunstancias similares. Definen como evaluación cognitiva al proceso de categorización de un evento según su efecto sobre el propio bienestar. Postulan que los eventos no son aversivos per se sino que lo son de acuerdo a la significación que el individuo les otorga.

Es limitada la literatura que relaciona efectividad con valoración de amenaza, por ejemplo Richaud y Sacchi (1999), realizaron un estudio sobre las variables moderadoras del estrés, para determinar la influencia del estilo atribucional, la confianza interpersonal y el apoyo social sobre la percepción de la amenaza. Su muestra estuvo conformada por 260 participantes de ambos sexos, con una edad promedio de 30 años de edad, de nivel socieoeconómico medio, residentes de la ciudad de Buenos Aires. Se les aplicó la Escala de Estilo Atribucional; la Manheim Interview of Social Support, la Escala de Confianza Interpersonal, y el Inventario de Situaciones y Respuestas de Ansiedad. Para estudiar las variables de interés se realizaron diversos análisis de perfiles. Con base en sus resultados sugieren que el apoyo social no tiene una influencia significativa sobre la percepción de amenaza. En lo que se refiere a la influencia del Estilo Atribucional consideran que las personas indefensas perciben más amenaza, especialmente expresada como ansiedad cognitiva y, en menor grado, como ansiedad motora o conductual. Por último, la confianza interpersonal demostró ser una variable importante en la percepción de amenaza. Los participantes que presentaron alta desconfianza en las personas de su entorno percibían un mundo más amenazante, que se manifestaba como ansiedad 
cognitiva, fisiológica y motora o conductual. Los autores concluyen que si bien existe una amenaza inherente a los sucesos aversivos físicos o psicosociales que nos ocurren continuamente, la respuesta a los mismos se ve modulada (acentuada o disminuida) en función de los recursos mencionados, especialmente en la confianza interpersonal y en el estilo atribucional, que determinan en cierta medida, la vulnerabilidad de la persona, es decir, el grado de amenaza.

McCrae (1984) realizó dos estudios para determinar la influencia de eventos de pérdida, amenaza, o desafío en la elección de estrategias de afrontamiento. En el primero de ellos, 225 hombres y mujeres completaron un cuestionario referente a sus respuestas de afrontamiento a un evento vivido recientemente, categorizado por el investigador como pérdida (muerte de un padre o amigo, divorcio o separación, robo); amenaza (enfermedad personal u hospitalización, pérdida de la visión o el oído, enfermedad en la familia, problemas para encontrar trabajo); o desafío (elección de la oficina, el comienzo de la escuela, ascenso laboral, matrimonio, embarazo o llegada del bebé). En el segundo estudio, 151 participantes completaron una versión corta del cuestionario en respuesta a tres estresores, pero esta vez ellos mismos seleccionaron el evento como pérdida, amenaza o desafío. En ambos estudios se encontró que un tipo de estresor tiene un efecto consistente y significativo en la elección de los mecanismos de afrontamiento. Los resultados revelaron una marcada diferenciación en la selección de los mecanismos apropiados a diferentes condiciones. Específicamente, indican que individuos enfrentados a eventos valorados por ellos mismos como amenazantes tienden a usar fe, fatalismo, y pensamientos de deseo, como mecanismos para reducir ansiedad, al reconstruir el evento o su resultado. Por otra parte sugiere que los mecanismos más inmaduros, tales como reacciones hostiles, fantasía, escape y pasividad no son usados bajo condiciones de amenaza. Añade que conductas específicas en situaciones concretas están determinadas por un enorme número de influencias, muchas de ella altamente idiosincrásicas.

Lahoz y Forns (2005), realizaron un estudio con el objetivo de analizar la valoración de las características de los estresores y su relación con la elección de las estrategias de afrontamiento empleadas para hacerles frente, así como con los índices de sintomatología presentados por una muestra de 38 inmigrantes adultos extracomunitarios residentes en Barcelona. Se emplearon dos instrumentos: el Inventario de Respuestas de Afrontamiento (CRI) (Moos, 1993), que analiza el bienestar psicológico a través de 4 escalas: síntomas somáticos, ansiedad-insomnio, disfunción social y depresión grave, y el Cuestionario de Salud General (GHQ) (Goldberg y Hillier 1979), que se compone de tres apartados: en el primero, se le solicita a la persona que exponga un problema o una situación difícil que haya tenido que afrontar durante los pasados 12 meses; en el segundo, se presentan 10 reactivos con 4 opciones de respuesta tipo Likert, que proporcionan información sobre cómo el individuo valora el problema descrito. Las 10 preguntas acerca de su valoración del problema se refieren a:

1. Experiencia: experiencias previas de resolución de un problema similar al presentado

2. Predictibilidad: conocimiento sobre la probabilidad de ocurrencia del problema

3. Inminencia: existencia de tiempo de preparación para afrontar el problema

4. Amenaza: pensar en el daño que puede causar el problema

5. Desafío: percepción del problema como un desafío

6. Responsabilidad personal de causar el problema

7. Causa ajena del problema

8. Beneficio: extraer algo positivo de hacer frente al problema

9. Actualidad: si el problema se ha solucionado o no

10. Estado general: en caso de haberse solucionado el problema, evaluación general de la situación. 
El último apartado del cuestionario contiene 48 reactivos para evaluar ocho tipos de respuestas de afrontamiento. Las primeras cuatro escalas miden el afrontamiento por aproximación y las cuatro últimas, el afrontamiento por evitación.

Los resultados mostraron que las características de los estresores que demuestran una relación con la salud son: la experiencia previa, la predictibilidad (grado en que el acontecimiento puede ser esperado), la percepción de amenaza, la sensación de no extraer nada positivo del hecho de hacer frente al estresor, y la actualidad del mismo. En cuanto a la elección de las estrategias de afrontamiento, hallaron que depende de la experiencia previa, la predictibilidad, la percepción de amenaza, de desafío, la valoración de externalidad del estresor, la falta de beneficio y la no resolución actual. Dichos autores afirman que: a) cuando la persona tiene menos experiencia para hacer frente una situación similar utilizará más estrategias de análisis lógico; y b) cuanta menos predicción de la ocurrencia del problema, menor será el uso de estrategias de reevaluación positiva, resolución de problemas y búsqueda de gratificaciones. Con respecto a la percepción de amenaza encontraron que correlacionó negativamente con la elección de estrategias de resolución de problemas y positivamente con una mayor presencia de síntomas de ansiedad e insomnio, a diferencia de la percepción de desafío que correlacionó con un mayor uso de estrategias de reevaluación positiva y de resolución de problemas. Sus resultados indican que cuando la causa del problema se percibe como externa a la persona, ésta recurre menos a estrategias de reevaluación positiva; que a menor beneficio percibido, menor es el uso de estrategias de reevaluación positiva, búsqueda de guía, resolución de problemas y búsqueda de gratificaciones alternativas; que cuando el problema no se ha resuelto, la persona utiliza menos estrategias de reevaluación positiva, evitación cognitiva y búsqueda de gratificaciones. Igualmente hallaron que cuando la persona no percibe ningún beneficio del hecho de afrontar el problema, presenta más síntomas somáticos, disfunción social depresión grave y malestar general. Por último, que cuando el problema no ha sido resuelto, hay un mayor número de síntomas somáticos y malestar general. Las personas de la muestra percibieron el problema como una amenaza, acentuando el aspecto nocivo del mismo, y su posible daño, por lo que presentaron una mayor sintomatología ansiosa e insomnio.

La conclusión de los autores es que existen determinadas características objetivas y subjetivas de los estresores que influyen en la salud de la muestra investigada, así como en la elegibilidad de las estrategias de afrontamientos puestas en marcha para afrontar dichos estresores.

Medina, Marcos, Pérez- Iñigo, Robles y Gómez- Trigo (2002), agregan que un mismo acontecimiento afecta a individuos diferentes, de distinta manera. Incluso, acontecimientos de características objetivas similares pueden afectar de manera distinta al mismo sujeto. Un mismo suceso puede ser percibido como un estímulo para el desarrollo personal o como un daño progresivo que se perpetúa crónicamente.

En el estudio presentado por Barraza (2006), con el objetivo de avanzar en la caracterización del estrés académico, se menciona que la relación de la persona con su entorno se ve mediada por un proceso cognoscitivo de valoración de las demandas del entorno y de los recursos internos necesarios para enfrentar estas demandas, que conduce necesariamente a la definición sobre la forma de enfrentarlo. Parte de que en la relación persona-entorno se presenta un componente que es la interpretación del acontecimiento vivido como peligroso, perjudicial o amenazante, independientemente de sus características objetivas.

De acuerdo con este autor, en el modelo transaccional del estrés la valoración puede ser de tres tipos: neutra, donde los acontecimientos no implican al individuo ni lo obligan a actuar; positiva, donde los acontecimientos son valorados como favorables para mantener el equilibrio y se cuenta con los recursos para actuar; y negativa, en donde los acontecimientos son valorados como una pérdida (falta de algo), una amenaza (daños o pérdidas que no han ocurrido pero que pueden ocurrir de forma inminente), un desafío (un reto potencialmente superable si se movilizan los recursos para ello), o simplemente se puede asociar a emociones negativas (miedo, ira, resentimiento). La persona realiza una valoración de las prácticas o acontecimientos que se constituyen en demandas de actuación para él, y de los recursos que dispone para enfrentarlos. Esta valoración puede tener 
dos resultados: cuando los acontecimientos o prácticas que se plantean como demanda pueden ser enfrentados con los recursos de que se dispone, se mantiene un equilibrio sistemático de relación con el entorno. Por otra parte, cuando los acontecimientos o prácticas que se plantean como demandas a la persona, no pueden ser enfrentados con los recursos de que se dispone y por lo tanto dicha demanda (ya en calidad de estímulo estresor) es valorada como una pérdida, una amenaza, o un desafío; sobreviene el desequilibrio sistémico en su relación con el entorno (situación estresante).

Scherer y Drumheller (1991), realizaron un estudio sobre la valoración cognitiva con jóvenes universitarios, quienes respondieron a un cuestionario de 16 reactivos para medir emociones asociadas con la valoración cognitiva de amenaza, pérdida, beneficio y desafío, concernientes a la evaluación académica, en tres períodos de tiempo (1 o 2 días antes de la evaluación, de 2 a 5 días después de la evaluación y de 3 a 5 días después de que las calificaciones fueron publicadas). Encontraron consistencia en la forma en la que los participantes valoran cognitivamente los eventos como estresantes, pero a mayor distancia del evento ocurrido y valorado como estresante, la relación era más débil, lo que les permite concluir que la valoración individual del significado de un evento es necesaria en la interpretación del grado en el que él o ella perciben que el evento puede ser estresante y la valoración puede ser operacionalizada en términos de emociones con respecto al evento.

Holahan y Moos (1990) evaluaron un conjunto de factores (depresión, eventos negativos de la vida, características personales, apoyo familiar), previamente identificados por su relación con la resistencia al estrés, en una muestra de 400 personas. Encontraron que los recursos de la persona pueden incrementar las aproximaciones de afrontamiento a través del proceso de valoración, fomentando creencias positivas de su propia habilidad para manejar exitosamente una experiencia amenazante.

Los estudios reseñados ilustran que el modelo transaccional es la piedra angular sobre la que descansa el estudio del fenómeno del estrés. Las bases de este modelo son cognoscitivas y representan una aproximación dualista con las implicaciones que han sido tratadas exhaustivamente por otros autores (Ryle, 1949; Ribes, 1982; Ribes y López, 1985; Ribes, 2001). Explicar el dualismo o las implicaciones que ha tenido en la conformación de nuestra disciplina es algo que rebasa con mucho el objetivo de este trabajo, baste decir que ha llevado, entre otras cosas, al planteamiento de pseudoproblemas y a la confusión conceptual. Aquí se parte de una concepción psicológica naturalista que se interesa en un replanteamiento conceptual de este fenómeno. Para esta labor, por supuesto, se reconocen los hallazgos y aportaciones de las perspectivas actuales en el estudio del estrés; sin embargo, la investigación que se presenta, se fundamenta en la idea de que algunos fundamentos teóricos y conceptuales surgidos en la perspectiva interconductual, permiten abordar el fenómeno sin aludir a un mundo cognoscitivo o mental.

Algunas de las premisas de esta aproximación de las que se parte para estudiar el llamado afrontamiento son:

1. Ribes (1990), con base en un conjunto de hallazgos (Moberg, 1985; Levine, 1985), plantea que el estrés corresponde a ciertos campos de contingencias más o menos bien delimitados, es decir, a ciertas situaciones que objetivamente generan la reacción emocional que las personas refieren como estrés; estas situaciones no contienen criterios de logro, es decir, no presentan demandas a satisfacer, resultados a obtener, o problemas a resolver. Sobre estas bases se puede afirmar que no todo lo que en el lenguaje cotidiano se refiere como estrés tiene que ver con este tipo de contingencias.

2. El ser humano se puede relacionar con dichas situaciones de diversas maneras que van desde una reacción biológica de activación, hasta la planeación de estrategias de solución en función de un sistema de creencias, esto es, existen distintos procesos de comportamiento cuya complejidad funcional es diferente (Ribes y López, 1985; Ribes, 1990), y ello no es razón para abordar los procesos más complejos como si fueran operaciones mentales o procesos cognoscitivos inconscientes.

3. Un mismo comportamiento puede analizarse a través de dimensiones distintas: La efectiva, que corresponde a las acciones, a lo que la gente hace para relacionarse con otra persona, situación o 
circunstancia; la afectiva, que tiene que ver con aquellos comportamientos que no alteran el ambiente sino que solamente afectan al propio individuo que los emite -percepción, atención, sensación, reacciones biológicas, emociones-; por último, la valorativa, en donde las personas se comportan reaccionando a elementos no presentes en la situación de estimulación a través de procesos sustitutivos de comportamiento (Ribes y López, 1985), esto es, se comportan en una situación como si estuvieran en otra (en aquélla en donde se explicitaron criterios o normas).

4. Un comportamiento complejo puede estar constituido por múltiples componentes y sus dimensiones pueden o no corresponder, dado que en algunos casos las dimensiones efectiva y afectiva están mediadas por la propia situación con la que se interactúa, mientras que la dimensión valorativa es necesariamente extrasituacional. (Corral, 2003).

El término afrontamiento, con el que actualmente se da cuenta de la manera en que una persona modula el estrés, es un concepto multívoco. Se incluyen en él: acciones, propensiones, creencias, entre otros; es decir, en ocasiones refiere componentes de un mismo comportamiento, en otras, alguna dimensión específica del mismo, o bien incluye disposiciones.

Entre otras cosas, el término afrontamiento alude a lo que Ribes (op.cit.) denomina 'competencias funcionales'. Este concepto tiene que ver con la capacidad de las personas para satisfacer una demanda, ajustarse a ella, resolver algún problema o producir cierto resultado; por supuesto en relación con situaciones que presentan una demanda a satisfacer. Se habla, por ejemplo, de estrategias de solución de problemas, lo que indica, por un lado, que la situación “estresora” demanda una acción específica, y por otro, que al demandar un resultado, no se trata de una contingencia objetivamente productora de estrés, sino de una contingencia de logro que demanda capacidad.

En el trabajo que aquí se presenta se parte de que cuando una persona refiere lo que se denomina reacciones de estrés en situaciones que contienen demandas de logro, lo que corresponde estudiar es su capacidad para satisfacer dichas demandas, es decir, sus competencias funcionales.

Para satisfacer las demandas que las diversas situaciones cotidianas pueden presentar es importante entender la relación que la persona establece con el ambiente, en este caso con la situación que contiene una demanda de logro, y estudiar tanto el nivel de complejidad funcional que la propia situación demanda para el comportamiento, como las diversas dimensiones y componentes del responder de la persona; el puro análisis morfológico de las respuestas o de la propia situación no permite dar cuenta de la complejidad del comportamiento a estudiar. Así por ejemplo, si se aproxima un animal peligroso cuando estamos distraídos y alguien nos grita "corre", la manera de satisfacer la demanda, es decir, el comportamiento que indicaría competencia o capacidad, es correr; y correr en una situación de esa naturaleza, es un comportamiento que puede tener diversos componentes y dimensiones. Se puede componer de reacciones biológicas de activación (dimensión afectiva del correr), de acciones, como el propio correr o gritar (dimensión efectiva del correr), y, en algunos casos, el correr puede depender de nuestras creencias, como cuando lo hacemos porque creemos que debemos obedecer instrucciones. En cada uno de los casos, aunque la morfología sea la misma -correr cuando alguien nos da la instrucción-, se puede tratar de procesos de comportamiento de diferente complejidad.

$\mathrm{Al}$ revisar la literatura sobre el tema, se puede notar que el estrés/afrontamiento se considera un solo fenómeno, y como parte de ese concepto se estudian y evalúan, entre otras cosas, diversas competencias para relacionarse con una serie de situaciones. Por ello, y en un intento de reformular el campo de estudio, creemos conveniente hacer esta primera distinción entre capacidades para enfrentar diversas situaciones de la vida ordinaria y reacciones que corresponden objetivamente a contingencias productoras de estrés. Igualmente vale la pena considerar que si en el conjunto de fenómenos que se agrupan en este campo de estudio, se incluyen capacidades para relacionarse con situaciones cotidianas, habría que evaluar factores que no han sido contemplados en otras perspectivas, tales como el nivel funcional del comportamiento demandado por una situación, el conjunto de habilidades que conforman una competencia específica, elementos definitorios de las diversas situaciones ambientales y la correspondencia o no correspondencia entre las dimensiones 
de los comportamientos que indican competencia. Es factible que al reformular el problema, se encuentre, por ejemplo que lo que se consideran diversas "estrategias de afrontamiento" no sean más que distintas dimensiones de un solo comportamiento que se encuentran reguladas por diferentes contingencias.

Con el propósito general de comprender este fenómeno a través de un conjunto de investigaciones, aquí se presenta el siguiente estudio, cuyo objetivo fue evaluar la posible correspondencia funcional entre la valoración de amenaza que refieren las personas con respecto a ciertas situaciones y su capacidad para satisfacer las demandas que dichas situaciones presentan, esto es, la correspondencia funcional entre la dimensión valorativa y la dimensión efectiva de algunas competencias.

\section{Método}

\section{Participantes.}

Participó una muestra intencional voluntaria de 347 participantes, de entre 15 y 70 años de edad, con una edad promedio de 29.6. De éstos, 233 eran mujeres y 114 hombres, 216 mencionaron ser solteros y 131 casados. En cuanto al nivel escolar reportado 16 participantes tenían estudios de posgrado, 9 alguna especialidad, 172 tenían licenciatura, 104 tenían el bachillerato, 34 la secundaria, y 12 solamente estudios de primaria.

\section{Muestreo.}

La selección de la muestra fue a través del procedimiento denominado "Muestreo por oportunidad", el cual consiste en seleccionar aquellos sujetos voluntarios y que presentan mayor oportunidad de ser seleccionados (Coolican, 1994).

\section{Tipo de estudio.}

El estudio se llevó a cabo a través de un diseño correlacional, dado que permite medir el grado de relación que existe entre las dos dimensiones de interés (Hernández, Fernández y Baptista, 1991).

\section{Variables e Instrumentos}

Variables de comparación: Género, nivel de competencia (alto y bajo) y contexto de desempeño.

Variables Dependientes. El grado de competencia en dos dimensiones: efectiva (hacer), y valorativa (creer). Dichas variables se evaluaron con las respuestas dadas a cada una de las situaciones planteadas en los distintos reactivos.

Instrumento: El instrumento consta de seis partes. La primera parte es para datos demográficos, la segunda contiene reactivos para explorar algunos datos relativos al estado de salud-enfermedad. Las competencias se evalúan en las siguientes cuatro partes del instrumento. Cada una de estas partes corresponde a un contexto de desempeño: escuela, trabajo, familia y pareja, e incluyen 27 reactivos en los que se solicitan tres distintas respuestas que corresponden a las tres dimensiones de las competencias evaluadas: efectiva, afectiva y valorativa. Nueve de los 27 reactivos que se incluyen para cada contexto evalúan la dimensión efectiva de las competencias, es decir, lo que hacen los participantes en cada situación; nueve la dimensión afectiva, sentimientos, sensaciones y reacciones de estrés; y los últimos nueve evalúan la dimensión valorativa, esto es, creencias con respecto a la situación; específicamente se evalúa si creen que la situación presentada es amenazante. Los reactivos presentan diversas situaciones que contienen demandas de logro y que fueron diseñados considerando contingencias caracterizadas por: logro o persistencia, toma de decisiones y flexibilidad al cambio. Los reactivos se responden con una escala tipo Likert que va de 'Nunca' a 'Siempre’. El número total de reactivos es de 108 (Rodríguez, Alatorre y Nava, 2008). 


\section{Procedimiento.}

Una vez sometido el instrumento a pruebas de validez y confiabilidad (Rodríguez, Alatorre y Nava, 2008), se procedió a aplicarlo a los 347 participantes. Dicha aplicación se llevó a cabo con cada uno de ellos de manera independiente. El investigador permaneció presente para dar instrucciones y resolver dudas. Una vez que el participante concluyó se agradeció por su colaboración y se prometieron informes acerca de los estudios realizados.

\section{Resultados}

Los resultados se analizaron con diversas pruebas estadísticas, aunque básicamente se empleó un análisis de correlación entre las variables de interés. Para empezar se presentan los descriptivos principales de las competencias en sus dimensiones efectiva y valorativa (Tabla 1). Aquí vale la pena tomar en cuenta que el puntaje más alto que podía obtenerse para cada dimensión fue de 216. En la tabla se observa que la media en la dimensión efectiva fue mayor que en la dimensión valorativa.

Tabla 1. Descriptivos generales de las dimensiones competenciales efectiva y valorativa.

\begin{tabular}{lcc}
\hline & EFECTIVA & VALORATIVA \\
MEDIA & 163.06 & 108.08 \\
MEDIANA & 167 & 107 \\
MODA & 186 & 72 \\
DESVIACIÓN E. & 29.51 & 28.25 \\
\hline
\end{tabular}

En la tabla 2 se presentan los descriptivos de la dimensión efectiva de las competencias considerando el contexto de desempeño. Se puede observar que los puntajes más altos se obtuvieron en el contexto escolar y los más bajos en el laboral.

Tabla 2. Descriptivos de competencias efectivas por contexto

\begin{tabular}{|l|c|c|c|c|}
\hline \multirow{2}{*}{} & CONTEXTO ESCOLAR & CONTEXTO LABORAL & CONTEXTO FAMILIAR & CONTEXTO DE PAREJA \\
\cline { 2 - 5 } & \multicolumn{4}{|c|}{ Dimensión Efectiva } \\
\hline Media & 42.88 & 38.85 & 39.51 & 41.81 \\
\hline Mediana & 44.00 & 42.00 & 41.00 & 44.00 \\
\hline Moda & 48.00 & 54.00 & 48.00 & 45.00 \\
\hline Desviación estandar & 7.85 & 12.30 & 9.58 & 9.26 \\
\hline
\end{tabular}

En la tabla 3 se presentan los mismos datos que la tabla anterior, pero para la dimensión valorativa. Se puede observar que la media más alta, como en el caso de la dimensión efectiva, se obtuvo para el contexto escolar, mientras que la más baja fue para las relaciones de pareja.

Tabla 3. Descriptivos de dimensión valorativa de las competencias por contexto

\begin{tabular}{|l|c|c|c|c|}
\hline \multirow{2}{*}{} & CONTEXTO ESCOLAR & CONTEXTO LABORAL & CONTEXTO FAMILIAR & CONTEXTO DE PAREJA \\
\cline { 2 - 5 } & \multicolumn{4}{|c|}{ Dimensión Valorativa } \\
\hline Media & $\mathbf{4}$ & 26.70 & 26.01 & 25.98 \\
\hline Mediana & 29.00 & 26.00 & 25.00 & 25.00 \\
\hline Moda & 27.00 & 18.00 & 17.00 & 18.00 \\
\hline Desviación estandar & 8.29 & 9.83 & 8.64 & 8.25 \\
\hline
\end{tabular}


Respondiendo al objetivo, para investigar la posible correspondencia funcional entre ambas dimensiones se aplicó una prueba de correlación de Pearson. Se encontró una correlación, aunque no fuerte, sí estadísticamente significativa $(\mathrm{r}=.215, \mathrm{p}<.01)$ entre las dos dimensiones competenciales: efectiva y valorativa. Con la finalidad de precisar esta relación se llevaron a cabo pruebas de correlación para cada uno de los contextos de desempeño evaluados. Se encontraron correlaciones estadísticamente significativas y más altas que la correlación en los puntajes totales, solamente en dos de los cuatro contextos de desempeño evaluados, en el contexto laboral $(r=.494, \mathrm{p}<.01)$ y en el familiar $(r=.411, \mathrm{p}<.01)$.

Habiendo obtenido estos datos, se evaluaron competencias en sus dos dimensiones tomando en cuenta el género de los participantes. La tabla 4 muestra las principales medidas de tendencia central. Como se puede notar la media es ligeramente mayor en el caso de los hombres, tanto para la dimensión efectiva de sus competencias como para la valorativa.

Tabla 4. Descriptivos de competencia por género

\begin{tabular}{|l|c|c|}
\hline & EFECTIVA & VALORATIVA \\
\hline \multicolumn{3}{|c|}{ HOMBRES } \\
\hline MEDIA & 163.06 & 108.08 \\
\hline MEDIANA & 167.00 & 107.00 \\
\hline MODA & 186.00 & 72.00 \\
\hline DESVIACIÓN E. & 29.51 & 28.25 \\
\hline \multicolumn{3}{|c|}{ MUJERES } \\
\hline MEDIA & 161.89 & 103.6 \\
\hline MEDIANA & 165.50 & 102 \\
\hline MODA & 188 & 95 \\
\hline DESVIACIÓN E. & 29.5 & 24.37 \\
\hline
\end{tabular}

La tabla 5 presenta los resultados obtenidos para la dimensión efectiva de las competencias considerando tanto el género de los participantes como el contexto de desempeño. Las medias más altas fueron las que se obtuvieron en el contexto escolar, seguidas del contexto de pareja y el familiar; en el contexto laboral se obtuvieron los puntajes más bajos. Hay que notar que en el caso de los hombres la media más baja se obtuvo en el contexto laboral, mientras que en el de las mujeres ésta se obtuvo en el contexto familiar.

Tabla 5. Descriptivos para la dimensión efectiva por género y contexto

\begin{tabular}{|l|c|c|c|c|}
\hline & $\begin{array}{c}\text { CONTEXTO } \\
\text { ESCOLAR }\end{array}$ & CONTEXTO LABORAL & $\begin{array}{c}\text { CONTEXTO } \\
\text { FAMILIAR }\end{array}$ & CONTEXTO DE PAREJA \\
\hline \multicolumn{5}{|c|}{ HOMBRES } \\
\hline MEDIA & 42.45 & 38.34 & 38.82 & 41.78 \\
\hline MEDIANA & 44 & 41 & 40 & 44 \\
\hline MODA & 47 & 40 & 48 & 84 \\
\hline DESVIACIÓN E. & 8.11 & 10.95 & 10.07 & 41.84 \\
\hline \multicolumn{7}{|c|}{ MUJERES } \\
\hline MEDIA & 43.09 & 38.87 & 39.85 & 44 \\
\hline MEDIANA & 44 & 42 & 41 & 45 \\
\hline MODA & 48 & 54 & 47 & 9.71 \\
\hline DESVIACIÓN E. & 7.74 & 12.94 & 9.34 & \\
\hline
\end{tabular}


La tabla 6 muestra los mismos datos que la tabla anterior, pero para la dimensión valorativa, como puede observarse las medias son superiores para ambos géneros en el contexto escolar, mientras que las medias inferiores se encuentran, para el caso de los hombres en las situaciones familiares y para el de las mujeres en el contexto de pareja.

Tabla 6. Descriptivos para la dimensión valorativa por contexto y por género

\begin{tabular}{|l|c|c|c|c|}
\hline \multirow{2}{*}{} & $\begin{array}{c}\text { CONTEXTO } \\
\text { ESCOLAR }\end{array}$ & CONTEXTO LABORAL & $\begin{array}{c}\text { CONTEXTO } \\
\text { FAMILIAR }\end{array}$ & $\begin{array}{c}\text { CONTEXTO DE } \\
\text { PAREJA }\end{array}$ \\
\cline { 2 - 5 } & \multicolumn{5}{|c|}{ HOMBRES } \\
\hline MEDIA & 27.80 & 25.52 & 24.34 & 24.94 \\
\hline MEDIANA & 27 & 25 & 17 & 18 \\
\hline MODA & 18 & 25 & 7.72 & 7.83 \\
\hline DESVIACIÓN E. & 8.01 & 8 & MUJERES \\
\hline \multicolumn{7}{|c|}{} \\
\hline MEDIA & 30.06 & 27.29 & 26.83 & 26.01 \\
\hline MEDIANA & 30 & 26.50 & 19 & 18 \\
\hline MODA & 26 & 18 & 8.96 & 8.47 \\
\hline DESVIACIÓN E. & 8.35 & 10.59 & \\
\hline
\end{tabular}

Se aplicaron pruebas de correlación con el propósito de precisar, considerando la variable género, las relaciones entre las dimensiones competenciales de interés. En primer lugar se aplicó un coeficiente de correlación de Pearson para evaluar la relación entre la dimensión efectiva y la valorativa. Para los hombres no se encontró ninguna relación estadísticamente significativa; sin embargo, para las mujeres se encontró una relación estadísticamente significativa entre estas dos dimensiones $(\mathrm{r}=.262, \mathrm{p}<.01)$. Este análisis se llevó a cabo también considerando el contexto de desempeño. Al incluir esta variable, se encontraron correlaciones estadísticamente significativas entre las dos dimensiones; en el caso de los hombres, en donde no se había hallado antes ninguna correlación significativa, se encontraron correlaciones moderadas para el contexto laboral ( $r=.429$, $\mathrm{p}<.01)$ y para el familiar $(\mathrm{r}=.437, \mathrm{p}<.01)$; y en el caso de las mujeres se encontraron correlaciones menores: para el contexto de pareja $(\mathrm{r}=.221, \mathrm{p}<.01)$, para el familiar $(\mathrm{r}=.399, \mathrm{p}<.01)$, y para el escolar $(\mathrm{r}=.517, \mathrm{p}<.01)$. Este análisis permitió concretar los resultados obtenidos al considerar solamente los contextos de desempeño.

Una vez habiendo evaluado los descriptivos principales por género y contexto, se formaron dos grupos con base en sus puntajes en cada dimensión, esto se hizo a partir de los datos obtenidos en la mediana, así se formaron los grupos de competencia alta y baja. La tabla 7 muestra el número de participantes competentemente bajos y altos para las dos dimensiones respectivamente, aunque hubo muy poca diferencia entre ambos grupos, se puede notar que hay un mayor número de participantes en el grupo de competencia baja para ambas dimensiones, y aún más participantes en la dimensión valorativa.

Tabla 7. Número de participantes en los grupos de competencia alta y competencia baja en las dimensiones competenciales efectiva y valorativa

\begin{tabular}{|c|c|c|}
\hline & EFECTIVA & VALORATIVA \\
\hline COMPETENCIA BAJA & 174 & 176 \\
\hline COMPETENCIA ALTA & 173 & 170 \\
\hline
\end{tabular}


Las tablas subsecuentes (8 y 9), muestran el número de participantes en los grupos de competencia alta y baja por contexto para cada dimensión. La tabla 8 muestra de manera específica la dimensión efectiva, se encontró un mayor número de participantes en el grupo de competencia baja en el contexto laboral y en el de pareja, mientras que el mayor número de participantes del grupo de competencia alta estuvo en los contextos relativos a la escuela y la familia. Por otra parte, en la tabla 9 se muestran estos mismos datos para la dimensión valorativa, encontrándose mayor número de participantes del grupo de competencia baja para el contexto escolar y mayor número de participantes del grupo de competencia alta en el contexto familiar.

Tabla 8. Participantes por grupos de competencia baja y alta por contexto de desempeño en la dimensión efectiva

\begin{tabular}{|l|c|c|c|c|}
\hline & CONTEXTO ESCOLAR & CONTEXTO LABORAL & CONTEXTO FAMILIAR & CONTEXTO DE PAREJA \\
\hline $\begin{array}{l}\text { COMPETENCIA } \\
\text { BAJA }\end{array}$ & 179 & 181 & 179 & 181 \\
\hline $\begin{array}{l}\text { COMPETENCIA } \\
\text { ALTA }\end{array}$ & 168 & 166 & 168 & 166 \\
\hline
\end{tabular}

Tabla 9. Participantes por grupos de competencia baja y alta por contexto desempeño en la dimensión valorativa

\begin{tabular}{|l|c|c|c|c|}
\hline & CONTEXTO ESCOLAR & CONTEXTO LABORAL & CONTEXTO FAMILIAR & CONTEXTO DE PAREJA \\
\hline $\begin{array}{l}\text { COMPETENCIA } \\
\text { BAJA }\end{array}$ & 183 & 181 & 179 & 181 \\
\hline $\begin{array}{l}\text { COMPETENCIA } \\
\text { ALTA }\end{array}$ & 164 & 165 & 168 & 166 \\
\hline
\end{tabular}

\section{Análisis de resultados}

Los resultados obtenidos con la prueba de correlación aplicada entre el puntaje total obtenido para cada una de las dimensiones competenciales, indican que hay una relación entre la dimensión efectiva de las competencias y la valorativa, y aunque dicha relación no es fuerte, el dato sugiere que las acciones que reportan llevar a cabo los participantes para cumplir las demandas presentadas en el instrumento no necesariamente están reguladas por las mismas contingencias que su comportamiento valorativo respecto de estas mismas situaciones.

Considerando que el puntaje máximo que podría obtenerse era de 216 y considerando también el número de participantes que configura los grupos de competencia alta y competencia baja, hay que señalar que los participantes de esta muestra no fueron altamente competentes en términos efectivos, es decir, no lograban satisfacer las demandas que se plantearon en las diversas situaciones muestreadas en el instrumento (163). Con respecto a la dimensión valorativa, los resultados sugieren que los participantes valoraban las situaciones presentadas como regularmente amenazantes (108.08).

En lo que se refiere a efectividad y contextos, aunque con diferencias poco significativas, los participantes respondieron con mayor efectividad en los contextos escolar y de pareja, y de manera ligeramente menos efectiva en los contextos de familia y laboral. Esto pudiera estar relacionado con la escolaridad de la muestra, ya que la mayor parte de los participantes tenía estudios de licenciatura. 
Ahora, algo que es importante es que, a pesar de que el contexto escolar es donde reportan mayor capacidad para resolver demandas, también reportan valorar las situaciones presentadas como más amenazantes, a diferencia del contexto de pareja en el que reportan efectividad y es el contexto en el que valoran las situaciones de una forma menos amenazante.

Los resultados sugieren también diferencias de género para las dos dimensiones, los hombres responden con un poco de mayor efectividad a las demandas planteadas, y a la vez las valoran como más amenazantes que las mujeres.

Al aplicar pruebas de correlación considerando el género, se encontró, con respecto al puntaje total obtenido para cada una de las dos dimensiones, que solamente hubo una correlación débil, aunque estadísticamente significativa, para las mujeres, esto es, su hacer y la forma en que valoran las situaciones presentadas tienen cierta correspondencia. Al intentar precisar la información e incluir, no solamente el género, sino los diversos contextos de desempeño, se encontraron más correlaciones. Aquí hubo relaciones positivas y estadísticamente significativas tanto en hombres como en mujeres. Para los primeros parece haber una correspondencia funcional entre las dos dimensiones en el contexto laboral y en el familiar. Para las mujeres en el escolar, el familiar y el de pareja. Cabe agregar que las medias nos indican que tanto hombres como mujeres son más efectivos en el contexto escolar, seguido por el contexto de pareja y el familiar y menos en las situaciones laborales, lo que podría estar relacionado con la edad promedio (29 años) en donde no se tiene gran experiencia para el trabajo. Las medias obtenidas para la dimensión valorativa en hombres y mujeres sugieren que ambos valoran las demandas escolares como las de mayor amenaza, mientras que el contexto de menor amenaza para los hombres es el relativo al de familia y para las mujeres es el de de pareja.

Con relación a la inclusión de grupos con base en su grado de competencia, como se había indicado, el mayor número de participantes se encuentra en el grupo de competencia baja en ambas dimensiones, es decir, tanto en su capacidad para resolver las demandas presentadas en el instrumento como en la forma en que valoraron dichas demandas, esto es, no las valoraron como amenazantes con mucha frecuencia.

\section{Discusión}

En primera instancia cabe mencionar que los datos sugieren que no existe una correspondencia funcional total entre las dos dimensiones estudiadas, lo cual está teóricamente previsto por la aproximación que fundamenta este estudio. Como se señalaba, la dimensión valorativa del comportamiento, en este caso del comportamiento que indica competencia o capacidad, implica necesariamente procesos extrasituacionales, creer algo con respecto de una situación, una circunstancia, el comportamiento de otra persona o del propio, implica responder a elementos no presentes en las condiciones de estimulación que conforman una situación concreta; y esta es una característica que no tiene necesariamente el comportamiento efectivo que se da en la misma situación.

La gente al valorar, se comporta como si estuviera en otra situación, en aquella en donde se explicitaron criterios, normas, o reglas como formas de comportamiento. Por otro lado, al actuar frente a una demanda que se debe satisfacer, sea ésta la producción de algún resultado, el ajuste a un cambio o la solución de un problema, las personas, dependiendo de su historia y de otra serie de factores, pueden responder directamente a los elementos presentes en la situación, o podrían actuar regulados por sus propias creencias (en cuyo caso habría una correspondencia funcional entre las dos dimensiones). Cabe aclarar que para que una persona sea competente debe satisfacer la demanda de la situación y ello implica que sus acciones efectivas deben corresponder funcionalmente a las demandas presentadas. Si la situación, por ejemplo, demanda que la persona abra una puerta con una herramienta, el comportamiento efectivo sería el que está regulado por tales condiciones, es decir, se requiere de un comportamiento situacional instrumental; sin embargo, en otras situaciones las demandas requieren de comportamientos que implican procesos extrasituacionales, por ejemplo, 
si hay que tomar una decisión que afecta a otras personas y la decisión debe estar regulada por conocimientos. De esta manera las competencias, en su dimensión efectiva, pueden implicar procesos situacionales o bien procesos extrasituacionales, considerando que las demandas de la situación pueden satisfacerse con respuestas vinculadas directamente a las condiciones de estimulación presentes, o bien a algún comportamiento extrasituacional. Habrá casos en que la satisfacción de una demanda dependa de ciertas creencias, y otros en donde los juicios o valoraciones no correspondan funcionalmente a las demandas presentadas. Esto quiere decir que un mismo comportamiento, dependiendo de la naturaleza funcional de su relación con el ambiente, puede ser lo suficientemente complejo como para que una de sus dimensiones esté regulada por ciertas contingencias y otra, por contingencias diferentes. Por ello es que puede o no existir una correspondencia funcional entre dimensiones.

Esta postura contrasta con la expuesta por Lazarus y Folkman (1986), quienes mencionan que el individuo realiza, como parte de un proceso cognitivo, una valoración de la demanda presentada, así como de su propia capacidad para afrontarla y que sus acciones están sujetas a dicha valoración.

Sobre estas bases la aproximación transaccional dio lugar al concepto de 'percepción de amenaza' que ha resultado clave para orientar diversas investigaciones y generar explicaciones del fenómeno llamado estrés. Algunos autores afirman, por ejemplo que la percepción de amenaza influye, no solamente en la producción de reacciones de estrés, sino en la elección de estrategias de afrontamiento. Igualmente que las personas enfrentadas a eventos que ellos mismos valoran como amenazantes, emplean como estrategia de afrontamiento la fe o el fatalismo. Esta 'percepción de amenaza' se concibe como el primer eslabón de un proceso cognoscitivo que se da en el inconsciente, y se asume que juega un papel causal tanto en las acciones o verbalizaciones de la persona, como en sus reacciones emocionales (McCrae, 1984; Richaud y Sacchi, 1999; Lahoz y Faros, 2005). En nuestra opinión la llamada percepción de amenaza puede referir diversos aspectos, puede tratarse de un componente afectivo de algún comportamiento, en caso de que sea un componente propiamente perceptual, o bien puede tratarse propiamente de una valoración o creencia con respecto de una situación. Los dos casos representan niveles de complejidad distintas (Kantor, 1969); sin embargo, al parecer, gran parte de lo que se investiga está más bien relacionado con creencias; y si esto es así, se ha estado estudiando una de las dimensiones del comportamiento llamado estrés, desde una perspectiva dualista, asignándole, además, un papel causal.

Los resultados encontrados en este estudio no tienen que ver con la asignación de funciones causales. No se puede pensar que las creencias con respecto de la situación son causa del comportamiento de “estrés”, porque constituyen una de sus dimensiones. La capacidad o incapacidad para relacionarse exitosamente con la misma situación tampoco puede ser causa de lo que se cree respecto de ella. Ahora, si entre ambas dimensiones no hay una correspondencia funcional total, parece conveniente explorar, por un lado, los factores que determinan las creencias de los participantes y por otro, los factores relacionados propiamente con la capacidad de la gente para relacionarse de manera exitosa con las situaciones que conforman su vida cotidiana.

Dadas las diferencias halladas al considerar los contextos de desempeño, valdría la pena considerar las demandas sociales más frecuentes en cada contexto, las habilidades que éstas implican y el conjunto de criterios o creencias que norman el comportamiento social. Ello permitiría capacitar a la gente para relacionarse exitosamente en sus diversos contextos.

Por último, hay que insistir en que las llamadas reacciones de estrés se reportaron en situaciones que implican logro o capacidad, y que no corresponden con contingencias genuinamente productoras de estrés. Estamos, entonces, abordando un comportamiento morfológicamente similar aunque funcionalmente distinto al de estrés y ello implica, entre otras cosas, la necesidad de considerar las exigencias del medio en términos de competencia. Por esto y por las consideraciones antes expuestas, creemos importante seguir investigando bajo esta perspectiva con el objeto de obtener una mayor comprensión de este fenómeno y darle después aplicaciones más eficientes en el manejo y prevención de enfermedad. 


\section{Referencias}

Barraza, M. (2006). Un modelo conceptual para el estudio del estrés académico. Revista Electrónica de Psicología, 9, 3. Disponible en: www.iztacala.unam.mx/carreras/psicologia/psiclin

Billings, A. G., Moos, R. H. (1981). The role of coping responses and social resources in attenuating the stress of life events. Journal of Behavioral Medicine, 4, 139- 157.

Brannon, L. y Feist, J. (2001). Definición y medición del estrés. Psicología de la salud. Madrid: Thompson Learning.

Caballo, V., Buela- Casal, G., y Carboles, J. (1993). Manual de Psicopatología y trastornos psiquiátricos. Madrid: Siglo XX!, Vol. 1.

Cassidy, T. (1999). Stress, cognition and health. London: Routledge.

Coolican, H. (1994). Métodos de investigación y estadística en Psicología. México: Manual Moderno.

Corral, V. (2003). ¿Mapas cognoscitivos o competencias ambientales?. En: E. Díaz-González y M. L. Rodríguez (comps.). Perspectivas sobre cognoscitivismo en psicología. México: Editorial FESI- UNAM.

Goldberg, D. P. \& Hiller, V. F. (1979). A scaled version of the General Health Questionnaire. Psychological Medicine. 9, 139 - 145.

Graig, E. (1993). Stress as a consequences of urban physical environment. En L. Golberger; S. Breznits (Eds.), Handbook of stress. Theoretical and clinical aspects. New York: The Free Press.

Hernández, R., Fernández, C. y Baptista, P. (1991). Metodología de la Investigación. México: McGraw-Hill.

Holahan, Ch. J. \& Moos, R. H. (1990). Life stressors, resistance factors, and improved psychological functioning: an extension of the stress resistance paradigm. Journal of Personality and Social Psychology. 58, 5, 909 - 917.

Kantor, J. R. (1969). Principles of Psychology. Chicago: Principia Press.

Lahoz, S. y Forns, S. (2005). Valoración de estresores, estrategias de afrontamiento y salud en una muestra de inmigrantes extracomunitarios. Revista Electrónica Psicología Científica. Disponible en: www.psicologiacientifica.com

Lazarus, R. y Launier, R. (1978). Stress- related transactions between person and enviroment. En L. A. Pervin y M. Lewis (Eds.) Perspectives in interactional psychology (pp. 287 - 327). Nueva York: Plenum.

Lazarus, R. S. y Folkman, S. (1984). Stress, appraisal and coping. New York: Springer Publishing Company.

Lazarus, R. S. y Folkman, S. (1986). Estrés y procesos cognitivos. Barcelona: Martínez Roca

Lazarus, R. (2006). Coping with anging. EUA: Oxford University Press.

Levine, S. (1985). A definition of stress? En G. P. Moberg (Ed.). Animal stress. Bethesda: American Physiological Society.

Levy-Levoyer, C. (1985). Psicología y medio ambiente. Madrid: Ediciones Morata.

McCrae, R. (1984). Situational determinants of coping responses: loss, threat, and challenge. Journal of Personality and Social Psychology, 4, 919-928. 
Medina, A., Marcos, G., Pérez- Iñigo, G., Robles, S., Gómez- Trigo, O. (2002). Variables implicadas y repsuesta de estrés en situaciones de amenaza. Revista Electrónica de Psicología Científica. Disponible en: www.psicologiacientifica.com

Moberg, G. P. (1985). Animal stress. Bethesda: American Physiological Society.

Moos, R. (1993). Coping Responses Inventory - Adult Form Professional Manual. Odesa, Fla. Psychological Assessment Resources.

Ribes, E. (1982). El Conductismo: reflexiones críticas. Barcelona: Fontanella.

Ribes, E. y López, F. (1985). Teoría de la conducta. Un análisis de campo y paramétrico. México: Trillas

Ribes, E. (1990). Psicología y Salud: Un análisis conceptual. España: Martínez Roca.

Ribes, E. (2001). Los conceptos cognoscitivos y el problema de la observabilidad. Acta Comportamentalia, 9, Monográfico, 9-19.

Rice, P. (1999). Stress and health. USA: Brooks/ Cole Publishing Company.

Ricchaud de Minzi, M. \& Sacchi, C. (1999). Variables moderadoras del estrés. Revista Latinoamericana de Psicología, 31, 002. Fundación Universitaria Konrad Lorenz. Bogotá, Colombia 335- 365. Disponible en: http://redalyc.uaemex.mx

Rodríguez, J. (1995). Estrés psicosocial y su afrontamiento. Psicología Social de la Salud.

Rodríguez, M. L.; Alatorre, L. y Nava, C. (2008). Confiabilización de un instrumento para evaluar competencias en diversos contextos de desempeño: un estudio preliminar. Revista Electrónica de psicología Iztacala, 11, 1, 78-101.

Ryle, G. (1949). The concept of mind. N. Y: Barnes and Noble.

Scherer, R. y Drumheller, P. (1991). Consistency in cognitive appraisal on a stressful event over time. The Journal of Social Psychology, 132 (4), 535-555.

Seyle, H. (1956) The stress of the life. University of Canada Montreal.

Seyle, H. (1936). A sindrome produced by diverse noxious agents. Nature, 2. 132-138. 